\title{
Evaluation of visual hallucination based on pareidolia testing in patients with Alzheimer's disease
}

Şadiye GÜMÜŞYAYLA ${ }^{1}$, Hesna BEKTAŞ ${ }^{1}$, Gülsüm AKDENIZ², Gönül VURAL ${ }^{1}$, Mehmet illker YÖN ${ }^{1}$

${ }^{1}$ Department of Neurology, Faculty of Medicine, Ankara Yildirim Beyazit University, Ankara, Turkey.

${ }^{2}$ Department of Biophysic, Faculty of Medicine, Ankara Yildirim Beyazit University, Ankara, Turkey.

\section{SUMMARY}

Pareidolia is a complex visual illusion that is considered as a marker of visual hallucinations. This study aimed to measure pareidolic illusions with a simple experimental paradigm using the pareidolia test as an indicator of visual hallucination in Alzheimer's disease.

A total of 34 patients with Alzheimer's disease and 30 healthy subjects with no difference in age and sex were included in the study. The degree of cognitive impairment was determined using the Mini-Mental State Examination and the Montreal Cognitive Assessment (MoCA) test. Sixty images were used as pareidolia test materials including human face, some objects, and artifacts. The patients' answers were divided into three groups as correct response, illusory response, and other responses while the test was scored.

The averages of healthy individuals in the correct response group were higher than those in the patient group. Moreover, the mean number of illusory responses in the patients was $2.27 \pm 3.42$, whereas no illusory response was obtained in healthy subjects. No relationship between the number of illusory responses and the Mini-Mental Test score, MoCA test score, and duration of illness was observed.

Pareidolia paradigm was a simple and useful test that revealed the tendency to subclinical visual hallucinations or hallucinations in patients with Alzheimer's disease.

Key words: Alzheimer disease, pareidolia, visual hallucination

\section{INTRODUCTION}

Alzheimer's disease (AD) is the most common form of dementia. It is a progressive neurodegenerative disease characterized by a decreased cognitive function, a reduced ability to maintain daily life activities, behavioral changes, and psychiatric symptoms. Aging is an important risk factor for AD, with the incidence doubling every 5 years after age 65 . The incidence and prevalence of $A D$ are increasing, attributable to genetic and environmental factors. In addition to the dominant finding of memory impairment (1-5), patients with AD may suffer from depression, agitation, delirium, and hallucinations. Visual hallucinations and illusions are behavioral symptoms that occur in several cognitive disorders (1, 2) and have been attributed to temporo-occipital cortical lesions $(2,4)$. Although both visual and auditory hallucinations occur in dementia, the former is more frequent $(4,5)$, with visual illusions classified as a minor form of hallucination $(2,3)$.

Visual hallucinations occur at a rate of $12 \%-53 \%(5)$ and are associated with rapid cognitive impairment $(6,7)$. Visual hallucinations occur with unequal intensity in all stages of $A D$, being most common during the middle phase $(8,9)$.

Pareidolia can be assessed by a simple neuropsychological test that also indicates a predisposition to subclinical or clinical visual hallucinations. Therefore, whether pareidolia occurs in patients with AD was investigated in this study.

\section{MATERIAL AND METHODS}

The study population consisted of 34 patients with $A D$ and 30 healthy controls. All 64 participants were treated at the Yildirim Beyazit University Faculty of Medicine and Ankara Atatürk Training and Research Hospital Neurology Clinic. AD was diagnosed according to the Diagnostic and Statistical Manual of Mental Disorders, Fourth Edition criteria. The age range of the patients with AD was 59-86 years and that of the healthy controls was $55-85$ years. Patients with AD and healthy individuals were 
matched in terms of age, sex, educational level, and visual acuity. The ethics committee of the Yıldırım Beyazıt University Faculty of Medicine Hospital approved this study. A detailed clinical evaluation of all study participants was conducted by researchers in a neurology outpatient setting. It included medical history, neurological examination, age, sex, and, for the patients with $A D$, the duration of illness and treatment modality. In addition, the Mini-Mental Test (MMT) and the Montreal Cognitive Assessment (MoCA) test were performed by experienced researchers, and the results were used to determine the cognitive status. The physical and neurological examinations of all healthy controls were normal. Patients with other progressive brain diseases, other chronic systemic diseases, or a history of chronic drug use were excluded from the study. None of the healthy controls in the control group had malignancy, systemic disease, neurological disease, or a history of chronic drug use. The occurrence of visual hallucinations and illusions was assessed in all participants, and all who indicated experiencing such hallucinations were excluded. A pareidolia test was performed to assess pareidolic illusions in the patient and control groups. The test consisted of 60 photographs of "faces" with neutral expressions (face-pareidolia images). Pareidolia and true face photographs were pretransformed bysmoothing and luminosity matching to equalize the reflected light intensity and tone of the images. Scrambled images of the faces and pareidolia photos, preprocessed for use in the study, were produced using MATLAB. For mixed images, face and face-pareidolia images were divided into a large number of squares that were subsequently randomly repositioned. The scrambled images were used for control purposes to determine whether the participants actually saw a face or a face-pareidolia image. The photographs included human faces, various objects, and "noisy" faces. The data were collected by researchers in one-on-one sessions with the participants. The participants were shown a total of 60 cards and asked to identify the face or face-pareidolia images. The answers were classified into three groups: (1) correct responses, in which the objects in the pictures were identified exactly and correctly; (2) illusory responses, in which the objects not in the pictures were identified; (3) other responses, in which either an answer was not provided or the image was not recognized at all.

\section{Statistical analyses}

Sex and other participant variables were reported as numbers and percentages. Means \pm standard deviation, median, interquartile range (IQR), and minimum and maximum values were calculated for descriptive statistics to inform the analysis of the response variables of age, MMT score, MoCA score, duration of illness, and pareidolia test result. The normality of the age and response variable data was tested using the Shapiro-Wilk test. The parametric tests were used to analyze the normalized parameters. Descriptive statistics were expressed as means \pm standard error. The nonparametric tests were used to analyze the normally distributed data. Independent two-sample t-tests were used to determine whether the difference in age between the patient and control groups was significant. The nonparametric Mann-Whitney $U$ test was used to determine statistically significant differences in responses in the pareidolia test between the patient and control groups. A chi-square test was used to determine the impact of sex on the responses of the patient and control groups. The Spearman rho correlation analysis was performed to assess the relationship between an illusory response and the MMT score, MoCA score, and disease duration. IBM SPSS Statistics for Windows software (version 21.0; IBM Corp., NY, USA) was used for all statistical analyses. A Pvalue $<0.05$ indicated statistical significance.

\section{RESULTS}

The mean age of the patient and control groups was $72.52 \pm$ 7.95 and $72.33 \pm 7.87$ years, respectively. The age and sex of the patients and healthy controls were not significantly different ( $P$ $=0.922$ and 0.959 , respectively). In the patient group, the mean and median MMT scores were $21.38 \pm 5.69$ and 24.00 (IQR, 6.25) and the mean and median MoCA scores were $17.97 \pm 6.49$ and 19.00 (IQR, 10.75), respectively. The mean and median duration of illness in the patient group was $2.61 \pm 1.37$ and 2.00 (IQR, 1.25) years (Table 1). The mean and median number of correct responses in the patient group was $27.44 \pm 3.50$ and 29.00 (IQR, 4.00). The median number of correct responses in the control group was 30.00 (IQR, 1.00), which was significantly different from that of the patient group ( $P=0.001)$. Regarding the illusory responses, the mean and median scores were $2.26 \pm 3.47$ and 0.50 , respectively, with the minimum score being 0 . In the control group, only one illusory response was present, such that the difference versus the patient group in the number of illusory answers was also significant $(P<0.001)$. For other responses, the mean and median scores of the patients were $0.29 \pm 0.52$ and $0(I Q R, 1.00)$, respectively. No significant difference was found between the patient and the control group ( $P=0.816)$ (Table 2).

The relationship between the illusory response values of the patients and their MMT scores, MoCA scores, and disease duration were not statistically significant (Table 3).

\section{DISCUSSION}

In this study, the hypothesis that subclinical visual hallucinations could be detected using the pareidolia test in patients with $A D$ was tested. The results showed that pareidolic illusions were more frequent in patients with $A D$ than in healthy controls. The latter 
TABLE 1: Descriptive statistics of study cohort.

\begin{tabular}{llll}
\hline Variables & Mean \pm SS & Median (IQR) & Min; Max \\
\hline Age & $72.52 \pm 7.95$ & $74.00(11.00)$ & $55 ; 86$ \\
MMT score & $21.38 \pm 5.69$ & $24.00(6.25)$ & $3 ; 25$ \\
MoCA score & $17.97 \pm 6.49$ & $19.00(10.75)$ & $3 ; 25$ \\
Disease duration & $2.61 \pm 1.37$ & $2.00(1.25)$ & $1 ; 7$ \\
Correct response & $27.44 \pm 3.50$ & $29.00(4.00)$ & $17 ; 30$ \\
Illusory response & $2.26 \pm 3.47$ & $0.50(3.25)$ & $0 ; 13$ \\
Other response & $0.29 \pm 0.52$ & $0(1.00)$ & $0 ; 2$ \\
\hline
\end{tabular}

MMT: Mini Mental Test; MoCA: Montreal Cognitive Assessment, IQR: Interquartile range

TABLE 2: Comparison of age and correct, illusory and 'other' responses between the patients and control groups.

\begin{tabular}{|c|c|c|c|c|}
\hline \multirow[t]{3}{*}{ Variable } & \multirow{3}{*}{$\begin{array}{l}\text { Patient group } \\
\text { Mean } \pm \text { SS } \\
\text { Median (IQR) }\end{array}$} & \multirow{3}{*}{$\begin{array}{l}\text { Control group } \\
\text { Mean } \pm \text { SS } \\
\text { Median (IQR) }\end{array}$} & \multicolumn{2}{|c|}{ Test Statistic } \\
\hline & & & $\mathrm{t} ; * \mathrm{Z}$ & $p$ \\
\hline & & & & \\
\hline Age & $72.52 \pm 7.95$ & $72.33 \pm 7.87$ & 0.099 & 0.922 \\
\hline Correct response & $29.00(4.00)$ & $30.00(1.00)$ & $* 3.238$ & 0.001 \\
\hline Illusory response & $0.50(3.25)$ & $0(1.00)$ & $* 4.415$ & $<0.001$ \\
\hline Other response & $0(1.00)$ & $0(1.00)$ & $* 0.233$ & 0.816 \\
\hline
\end{tabular}

TABLE 3: Correlation between MMT skore, MoCA score, disease duration and number of illusory responses.

\begin{tabular}{lll} 
Variables & Spearman Rho Relation Coefficient & $p$ \\
\hline Illusory response - MMT score & -0.183 & 0.300 \\
Illusory response - MoCA score & -0.148 & 0.404 \\
Illusory response - Disease duration & 0.318 & 0.067
\end{tabular}

MMT: Mini Mental Test, MoCA: Montreal Cognitive Assessment

also had a significantly higher number of correct responses than the patients with AD. Thus, although none of the patients with AD had visual hallucinations, subclinical hallucinations were detected by the pareidolia test.

Visual hallucinations and pareidolias were persistent phenomena with similar mechanisms. However, because patients with cognitive decline rarely mention visual hallucinations to clinicians, the use of visual perception-based instruments to exclude subclinical hallucinations is an important part of the evaluation of patients with AD $(1,2)$. Studies in patients with Parkinson's disease and Lewy body dementia have shown that the pareidolia test can detect otherwise clinically undetected/unreported hallucinations $(1,2,10)$.

Pareidolias are complex visual illusions perceived as meaningful objects and are considered equivalent to visual hallucinations (2). Similar to visual hallucinations, pareidolias result in things being seen that are not actually present when the individual is awake, with the eyes open. Pareidolias represent the brain's attempt to interpret what is seen based on what has been learned before, even though the two may be unrelated. Tests of pareidolia measure illusions, such as visual hallucinations, and are valid for use in healthy individuals as well as in patients with Lewy body dementia and AD. Such tests have also been used to differentiate the latter two diseases $(1,2)$. Furthermore, pareidolia studies can provide insights into how the brain combines sensory-stimulus inputs (bottom-up) and cognitive regulation (top-down) processing of these inputs. Face pareidolia is the perception of illusionary faces. It is the most well-known type of pareidolia because of the social importance of faces and the ability of people to recognize them. In studies using face recognition tests, activity in the visual cortex was shown to be related to the specific phenomenological characterization of visual hallucinations.

Pareidolia tests were used in patients with AD in this study because they reveal subclinical visual hallucinations. The results showed that those without a history of hallucinations often provided an 
illusory response during the test. In contrast, no association was found between illusory responses and MMT and MoCA scores, denoting cognitive status. In a study conducted in patients with Parkinson's disease, illusory responses correlated with pareidolia test results and MMT scores (1). This likely reflected posterior cortical dysfunction, as previous studies demonstrated a correlation between bilateral temporal, parietal, and occipital hypometabolism and the number of illusory responses. Moreover, a single-photon emission computed tomography (SPECT), positron emission tomography, and volumetric magnetic resonance imaging (MRI) studies have shown a relationship between postural cortical dysfunction and visual hallucination in patients with Parkinson's disease and Lewy body dementia (2, 11-15). Visual hallucinations in $A D$ usually develop just before the onset of the advanced stage, suggesting a critical level of deterioration as a prerequisite, such as hallucinations. However, other factors are also likely to be involved because patients with $A D$ can have hallucinations during both the early and advanced stages (16).

Patients with AD show specific areas of temporal and parietal hypoperfusion on functional brain imaging $(17,18)$ as well as parietal hypoperfusion on SPECT studies (19). In functional MRI studies, hallucination was found to correlate with frontal hypometabolism or frontal and temporoparietal hypometabolism (20). Occipital volume was shown to be smaller than the volume of the entire brain of individuals without visual hallucinations (21). Thus, visual hallucinations in $A D$ could be instructive for identifying the brain regions that have lost function.

In summary, the frequency of subclinical visual hallucinations in patients with $A D$ was investigated, and evidence of visual hallucinations was detected in a significant proportion of these patients. Similar studies might be informative in terms of $A D$ progression. However, this study also had several limitations. First, the number of patients was small and the brain imaging findings of the patients with $A D$ could not be meaningfully analyzed in the context of the results of the pareidolia test. Studies of patients with suspected visual hallucinations employing functional imaging methods would therefore be informative.

\section{ACKNOWLEDGMENTS}

The authors would like to thank the Textcheck Scientific and Technical Editing Service for their professional editing. Hatice Hilal Aktaş carried out the statistical research.

\section{REFERENCES}

1. Uchiyama M, Nishio Y, Yokoi K, Hirayama K, Imamura T, Shimomura T, Mori E. Pareidolias: complex visual illusions in dementia with Lewy bodies. Brain 2012;135:2458-2569.
2. Uchiyama M, Nishio Y, Yokoi K, Hosokai Y, Takeda A, Mori E. Parkinsonism Relat Disord. Pareidolia in Parkinson's disease without dementia: A positron emission tomography study. 2015;21(6):603-609.

3. Fenelon G, Mahieux F, Huon R, Ziegler M. Hallucinations in Parkinson's disease: prevalence, phenomenology and risk factors. Brain 2000;123(Pt 4):733-745.

4. Muller AJ, Shine JM, Halliday GM, Lewis SJ. Visual hallucinations in Parkinson's disease: theoretical models. Mov Disord 2014;29:15911598.

5. Patterson MB, Schnell AH, Martin RJ, Mendez MF, Smyth KA, Whitehouse PJ. Assessment of behavioral and affective symptoms in Alzheimer's disease. J Geriatr Psychiatry Neurol 1990;3(1):21-30.

6. Lopez OL, Wisniewski SR, Becker JT, Boller F, DeKosky ST. Psychiatric medication and abnormal behavior as predictors of progression in probable Alzheimer disease. Arch Neurol 1999;56(10):1266-1272.

7. Magni E, Binetti G, Bianchetti A, Trabucchi M. Risk of mortality and institutionalization in demented patients with delusions. J Geriatr Psychiatry Neurol 1996;9(3):123-126.

8. Drevets WC, Rubin EH. Psychotic symptoms and the longitudinal course of senile dementia of the Alzheimer type. Biol Psychiatry 1989;25(1):39-48.

9. Reisberg B, Ferris SH, de Leon MJ, Crook T. The Global Deterioration Scale for assessment of primary degenerative dementia. Am J Psychiatry 1982;139(9):1136-1139.

10. Yokoi K, Nishio Y, Uchiyama M, Shimomura T, Iizuka O, Mori E. Hallucinators find meaning in noises: pareidolic illusions in dementia with Lewy bodies. Neuropsychologia 2014;56C:245-254.

11. Nagahama Y, Okina T, Suzuki N, Matsuda M. Neural correlates of psychotic symptoms in dementia with Lewy bodies. Brain 2010;133:557-567.

12. Boecker $\mathrm{H}$, Ceballos-Baumann AO, Volk D, Conrad B, Forstl H, Haussermann P. Metabolic alterations in patients with Parkinson disease and visual hallucinations. Arch Neurol 2007;64:984-988.

13. Perneczky R, Drzezga A, Boecker H, F€orstl H, Kurz A, Haussermann P. Cerebral metabolic dysfunction in patients with dementia with Lewy bodies and visual hallucinations. Dement Geriatr Cogn Disord 2008;25:531-5388.

14. Imamura $T$, Ishii $K$, Hirono $N$, Hashimoto M, Tanimukai S, Kazuai $\mathrm{H}$, et al. Visual hallucinations and regional cerebral metabolism in dementia with Lewy bodies (DLB). Neuroreport 1999;10:1903-1907.

15. Goldman JG, Stebbins GT, Dinh V, Bernard B, Merkitch D, deToledoMorrell $L$, et al. Visuoperceptive region atrophy independent of cognitive status in patients with Parkinson's disease with hallucinations. Brain 2014;137:849-859.

16. Ishioka T, Hirayama K, Hosokai Y, Takeda A, Suzuki K, Nishio Y, et al. Illusory misidentifications and cortical hypometabolism in Parkinson's disease. Mov Disord 2011;26:837-843.

17. Reiman EM, Caselli RJ, Chen K, Alexander GE, Bandy D, Frost J. Declining brain activity in cognitively normal apolipoprotein $E$ epsilon 4 heterozygotes: A foundation for using positron emission tomography to efficiently test treatments to prevent Alzheimer's disease. Proc Natl Acad Sci USA 2001;13;98(6):3334-3339.

18. Small GW, Ercoli LM, Silverman DH, Huang SC, Komo S, Bookheimer SY, Lavretsky H, Miller K, Siddarth P, Rasgon NL, Mazziotta JC, Saxena S, Wu HM, Mega MS, Cummings JL, Saunders AM, PericakVance MA, Roses AD, Barrio JR, Phelps ME. Cerebral metabolic and cognitive decline in persons at genetic risk for Alzheimer's disease. Proc Natl Acad Sci USA 2000; 23;97(11):6037-6042.

19. Kotrla KJ, Chacko RC, Harper RG, Jhingran S, Doody R. SPECT findings on psychosis in Alzheimer's disease. Am J Psychiatry 1995;152(10):1470-1475.

20. Grady CL, Haxby JV, Schapiro MB, Gonzalez-Aviles A, Kumar A, Ball MJ, Heston L, Rapoport SI. Subgroups in dementia of the Alzheimer type identified using positron emission tomography. J Neuropsychiatry Clin Neurosci 1990;2(4):373-384.

21. Holroyd S, Shepherd ML, Downs JH 3rd. Occipital atrophy is associated with visual hallucinations in Alzheimer's disease. J Neuropsychiatry Clin Neurosci 2000;12(1):25-28. 